\title{
Vertebral, intraspinal and other organ anomalies in congenital scoliosis
}

\author{
S. P. Mohanty ${ }^{1} \cdot$ Madhava Pai Kanhangad $^{1}$ (1) · Jayakrishnan K. Narayana Kurup ${ }^{1} \cdot$ Sibin Saiffudeen ${ }^{1}$
}

Received: 12 January 2020 / Revised: 12 March 2020 / Accepted: 2 May 2020 / Published online: 16 May 2020

(c) The Author(s) 2020

\begin{abstract}
Aims This study was undertaken to describe the pattern of vertebral, intraspinal and other organ anomalies in patients with congenital scoliosis and to determine the correlation between them.

Methods Complete medical and radiological records of 227 consecutive patients with congenital scoliosis were analysed. The radiographs were examined for type of vertebral anomaly, location and severity of deformity. The median curve progression index (MCPI) was calculated in 198 patients. The magnetic resonance imaging (MRI) of the whole spine was analysed to detect the presence of cord abnormalities. The presence of other organ-system anomalies was also noted. The independent sample $t$ test was used to compare severity of deformity between those with and without cord anomalies. The Chi-square test was used to compare frequency of cord abnormalities in different vertebral and organ-system anomalies.

Results Hemivertebra with contralateral bar had the highest MCPI, while block vertebrae and wedge vertebrae had the lowest MCPI. Forty-eight patients had 83 cord anomalies. There was no statistically significant difference in severity of deformity, between those with and without cord anomalies. Failure of segmentation had the highest frequency of cord anomalies $(p=0.01)$. There was no significant difference in the frequency of cord anomalies between those with and without other organ defects.

Conclusion Curve progression can be predicted by the underlying vertebral abnormalities. However, it cannot predict cord and other organ-system anomalies. Thus, all patients with congenital scoliosis must undergo MRI of the spine, electro- and echocardiography and ultrasonography of the abdomen to detect occult abnormalities and optimize the patient prior to deformity correction.
\end{abstract}

Keywords Congenital scoliosis $\cdot$ Vertebral anomalies $\cdot$ Intraspinal anomalies $\cdot$ Magnetic resonance imaging

\section{Introduction}

Around 8 weeks of intrauterine life, the bony elements of the spine begin to form and the neuraxis completes its infolding, to form the neural tube [1]. Any event during this period can lead to abnormalities of the spinal column as well as the spinal cord. Abnormalities of the bony elements present as failure of formation, failure of segmentation or both in the form of mixed anomalies. These abnormalities cause an imbalance in the longitudinal growth of the spine resulting in congenital spinal deformity. Multiple fused ribs when

Madhava Pai Kanhangad

madhava.pai@manipal.edu; kmadhavapai@gmail.com

1 Division of Spine Surgery, Department of Orthopaedics, Kasturba Medical College, Manipal Academy of Higher Education, Manipal, Karnataka 576-104, India present with bony bars on the concave side may result in rapid curve progression. Even though the contributing vertebral anomalies are present since birth, the deformity may only be evident later in childhood. The close embryological relationship between the spinal column and the spinal cord can result in concomitant intraspinal abnormalities such as split cord malformation (SCM), tethered cord, low-lying conus, syringomyelia and intraspinal masses [2-4].

Congenital spinal deformities are relatively rigid, may progress rapidly and when associated with intraspinal abnormalities have a higher risk of neurological complications. Being an embryological anomaly, it is often associated with genitourinary, cardiovascular, musculoskeletal and other abnormalities. These factors play an important role in the decision-making and management of congenital spinal deformities [2-7]. 
Several studies have been done to determine the pattern and presentation of congenital scoliosis, frequency of associated intraspinal and organ defects. The frequency of intraspinal anomalies ranged from 15 to $58 \%$. However, most of these studies were carried out on patients treated surgically, and few have been done to correlate them with vertebral anomalies [6-11]. This study was undertaken to describe the pattern of vertebral, intraspinal and other organ anomalies in 227 consecutive patients with congenital scoliosis and to determine the correlation between them.

\section{Materials and methods}

In a retrospective study, 582 patients who presented between January 2010 and June 2018 with spontaneous onset scoliosis before the age of 18 years, whose complete medical and radiographic records were available, were analysed. Clinical examination was done to detect neurological deficit, features of occult spinal dysraphism and presence of other congenital abnormalities. Standardized standing anteroposterior (AP), lateral and supine-stretch AP radiographs were obtained in all patients. Biochemical and haematological investigations were done to rule out infective and systemic disorders. All patients underwent magnetic resonance imaging (MRI) of the spine to detect craniovertebral and cord anomalies. Three hundred and thirty-seven patients with idiopathic $(n=296)$, presumed idiopathic with craniovertebral or spinal anomalies $(n=25)$, neuromuscular including syndromic scoliosis, tumours and previously operated cases $(n=21)$ were excluded. Thus, 227 patients with spinal deformity due to congenital vertebral anomalies formed the basis of this study. All patients underwent ultrasonography of the abdomen, electro- and echocardiography to detect renal and cardiac abnormalities. Institutional ethical committee clearance was obtained prior to the commencement of the study. One hundred and fifty-five patients underwent posterior spinal instrumentation, and fusion, of whom 29 underwent surgery within 1 year of presentation. The rest were followed up until skeletal maturity for curve decompensation.

\section{Radiological evaluation}

The digitalized radiographs were analysed by two independent observers in a blinded fashion, using Radiant version 4.6.5. In case of difference in the two readings, the decision of the senior author was considered to be final. The vertebral anomalies were classified according to Hedequist and Emans [12]. Location of the abnormality was recorded, and curve magnitude was measured using the method described by Cobb et al. [13]. In patients with concomitant kyphosis, the sagittal alignment was measured using the modified Konstam's angle [14]. The median curve progression index (MCPI) was calculated in 198 patients with follow-up greater than one-year prior to surgical intervention, using the method described by McMaster et al. [2].

\section{Evaluation of intraspinal anomalies}

MRIs of the whole spine including the craniovertebral junction were analysed to detect the presence of SCM, tethered cord, low lying conus, syringomyelia or any intradural masses. SCM was classified as type I (diastematomyelia with two hemicords in two dural sacs with bony or cartilaginous septum) and type II (two hemicords in a single dural sac), as described by Pang et al. $[15,16]$. The location and length of the split were also noted. In syringomyelia, the T1- and T2-weighted sagittal and axial sections of the MRI were analysed. The location of syrinx, length of the syrinx (number of vertebral segments traversed) and anteroposterior diameter of the syrinx at the level of maximum expansion were noted. The syrinx-cord ratio (SCR) was calculated by dividing the maximal syrinx diameter by the diameter of the cord at the same level. The syrinxes were classified as small ( $\mathrm{SCR}=0.2-0.25)$, medium $(\mathrm{SCR}=0.25-0.5)$ and large $(\mathrm{SCR}>0.5)[17,18]$.

\section{Statistical analysis}

Statistical Package for Social Science for Windows version 20 was used for statistical analysis. The intraclass correlation coefficient and kappa coefficient were used to determine inter- and intraobserver variability. Quantitative variables such as frequency of vertebral and cord anomalies were expressed as percentage. Continuous variables such as age, Cobb angle, extent of split cord and syrinx were expressed as mean $\pm \mathrm{SD}$. The one-way repeated ANOVA was used to compare Cobb's angle at the time of presentation in different vertebral anomalies. The independent sample $t$ test was used to compare age and Cobb's angle at presentation between those with and without cord anomalies. The Chi-square test was used to compare frequency of cord and other organ abnormalities in different vertebral anomalies. A $p$ value of less than 0.05 was considered to be significant.

\section{Results}

The inter- and intraobserver variation ranged from 0.85 to 0.88 . The descriptive statistics of the studied sample are shown in Table 1. The mean age of presentation was $8.8 \pm 5.2$ years, and girls were more commonly affected $(62.5 \%)$. Majority of the curves were located in the thoracic spine $(48.5 \%)$ followed by thoracolumbar spine $(31.8 \%)$. Right convex curves (53\%) were more common than the left convex curves. Failure of formation was the most common 
Table 1 Descriptive statistics of study population

\begin{tabular}{|c|c|c|c|c|c|c|c|}
\hline & \multicolumn{3}{|c|}{ Failure of formation $(n=144)$} & \multicolumn{3}{|c|}{ Failure of segmentation $(n=38)$} & \multirow{2}{*}{$\begin{array}{l}\text { Mixed } \\
\text { anomalies } \\
(n=45)\end{array}$} \\
\hline & $\operatorname{SHV}(n=61)$ & $\operatorname{MHV}(n=58)$ & $\mathrm{WV}(n=25)$ & $\mathrm{BV}(n=11)$ & UUB $(n=9)$ & $\mathrm{UUB}+\mathrm{CHV}(n=18)$ & \\
\hline Age of presentation & $9 \pm 1.3$ & $7.4 \pm 2.6$ & $10.3 \pm 1.46$ & $10.6 \pm 1.5$ & $9.1 \pm 2.3$ & $7.2 \pm 3.6$ & $9.4 \pm 2.7$ \\
\hline Gender (male/female) & $26: 35$ & $24: 34$ & $11: 14$ & $6: 5$ & $1: 2$ & $5: 13$ & $22: 23$ \\
\hline Mean Cobb's angle (coronal) & $38.7 \pm 1.6$ & $44.6 \pm 4.6$ & $23 \pm 2.5$ & $27 \pm 1.5$ & $45.6 \pm 2.7$ & $49.6 \pm 3.1$ & $41.2 \pm 3.5$ \\
\hline
\end{tabular}

SHV single hemivertebra, $M H V$ multiple hemivertebra, $W V$ wedge vertebra, $B V$ block vertebra, $U U B$ unilateral unsegmented bar, UUB $+\mathrm{CHV}$ unilateral unsegmented bar with contralateral hemivertebra

vertebral anomaly $(64.7 \%)$, followed by mixed anomalies $(18.2 \%)$, and failure of segmentation was the least frequent vertebral anomaly $(17.2 \%)$. The commonest vertebral anomaly was hemivertebrae $(n=119)$, and it constituted $82.6 \%$ of cases with failure of formation. Thirteen patients had concomitant kyphosis, six of which were due to failure of formation, two were due to failure of segmentation and five were due to mixed anomalies. The mean Cobb angle at the time of presentation was 41 degrees. The mean modified Konstam's angle at the time of presentation was $43^{\circ}$. The curve magnitude at the time of presentation was significantly lower in patients with block vertebrae and wedge vertebrae $(p<0.01)$. Patients with hemivertebra with contralateral bar in the thoracic spine had the highest MCPI (12.3\%/year) followed by the same lesion in the thoracolumbar spine (MCPI $=8 \%$ year). Block vertebrae and wedge vertebrae had the lowest MCPI ( $<2 \%$ year). The detailed MCPI based on vertebral level and type of anomaly is shown in Table 2.

The comparison between patients with and without cord anomalies is shown in Table 3. The Chi-square test demonstrated a statistically significant difference in the frequency of cord anomalies, with failure of segmentation having the highest frequency $(39.8 \%, p=0.01)$. Forty-eight patients $(21.2 \%)$ had 83 cord anomalies with $50 \%$ of them having multiple anomalies. Twenty-four of these patients underwent neurosurgical intervention 3 to 6 months prior to the deformity correction. The details of cord anomalies and their relationship with vertebral anomalies are shown in Table 4. SCM was the most frequently seen cord anomaly (64.6\%). Type 2 SCM $(74.2 \%)$ was more common than type 1 SCM. The mean extent of the split was $2.8 \pm 0.4$ levels, with type 1 SCM having greater extent of the split compared to type 2 SCM $(4.6 \pm 1.5$ vs $2.2 \pm 0.2)$. Forty-two percent of patients with SCM had concomitant cord anomalies, with syringomyelia being the most frequent. Syringomyelia was the second most common cord anomaly, and $63.2 \%$ had other cord anomalies. The mean extent of the syrinx was $4.6 \pm 0.3$ levels, and the mean SCR was $0.34 \pm 0.04$. Most of the syrinxes were small $(42.1 \%)$ to medium $(42.1 \%)$ in size and were present in the thoracic region $(57.9 \%)$ followed by the cervicothoracic region $(15.8 \%)$. All cases of tethered cord were associated with a low-lying conus. The level of the

Table 2 Details of median curve progression index at different vertebral levels and anomalies

\begin{tabular}{|c|c|c|c|c|c|c|c|c|}
\hline \multirow{3}{*}{$\begin{array}{l}\text { Vertebral level } \\
(n=198)\end{array}$} & \multicolumn{7}{|c|}{ Median curve progression index (degree/year) $(n=198)$} & \multirow{3}{*}{$\begin{array}{l}\text { Median curve } \\
\text { progression index at } \\
\text { each vertebral level }\end{array}$} \\
\hline & \multicolumn{3}{|c|}{ Failure of formation $(n=128)$} & \multicolumn{3}{|c|}{ Failure of segmentation $(n=34)$} & \multirow{2}{*}{$\begin{array}{l}\text { Mixed } \\
\text { anomalies } \\
(n=36) \\
(n=36)\end{array}$} & \\
\hline & SHV $(n=54)$ & $\operatorname{MHV}(n=49)$ & $\mathrm{WV}(n=25)$ & $\mathrm{BV}(n=11)$ & UUB $(n=9)$ & $\begin{array}{l}\mathrm{UUB}+\mathrm{CHV} \\
(n=14)\end{array}$ & & \\
\hline $\begin{array}{l}\text { Cervicothoracic } \\
\quad(n=18)\end{array}$ & 1.5 & 2.1 & - & 1 & 2.8 & - & 3.1 & 2.1 \\
\hline Thoracic $(n=96)$ & 5 & 5.9 & 2.5 & 1.1 & 4.1 & 12.3 & 3.4 & 5.9 \\
\hline $\begin{array}{l}\text { Thoracolumbar } \\
(n=63)\end{array}$ & 2.5 & 4.2 & 1 & 1 & 4.2 & 8 & 5.6 & 4.2 \\
\hline Lumbar $(n=21)$ & 1 & 1.9 & 1.2 & 1.3 & 2.8 & - & 4.3 & 1.9 \\
\hline $\begin{array}{l}\text { Median curve pro- } \\
\text { gression index } \\
\text { of each vertebral } \\
\text { anomaly }\end{array}$ & 2.5 & 4.1 & 0.7 & 0.6 & 3.5 & 5.2 & 3.3 & \\
\hline
\end{tabular}

$S H V$ single hemivertebra, $M H V$ multiple hemivertebra, $W V$ wedge vertebra, $B V$ block vertebra, $U U B$ unilateral unsegmented bar, UUB $+\mathrm{CHV}$ unilateral unsegmented bar with contralateral hemivertebra 
Table 3 Comparison between cases with cord anomalies and those without cord anomalies

\begin{tabular}{|c|c|c|c|}
\hline & $\begin{array}{l}\text { Cord anomaly present } \\
(n=48)\end{array}$ & $\begin{array}{l}\text { Cord anomaly absent } \\
(n=179)\end{array}$ & Comparison \\
\hline Age of presentation & $10.02 \pm 3.1$ & $9.52 \pm 4.9$ & $p=.42$ \\
\hline Cobb's angle at presentation & $40.86 \pm 2.31$ & $41.06 \pm 3.45$ & $p=.74$ \\
\hline \multicolumn{4}{|l|}{ Vertebral anomalies } \\
\hline Failure of formation & $25(17.4 \%)$ & $119(82.6 \%)$ & $p=.01$ \\
\hline Failure of segmentation & $15(39.5 \%)$ & $23(60.5 \%)$ & \\
\hline Mixed & $8(17.8 \%)$ & $37(82.2 \%)$ & \\
\hline Other organ anomalies & $13(27.1 \%)$ & $28(15.6 \%)$ & $p=.06$ \\
\hline
\end{tabular}

Table 4 Descriptive data of patients with cord anomalies $(n=48)$

\begin{tabular}{|c|c|c|c|c|c|}
\hline & Cord anomaly/vertebral anomaly & $\begin{array}{l}\text { Failure of formation } \\
(n=25)\end{array}$ & $\begin{array}{l}\text { Failure of segmenta- } \\
\text { tion }(n=15)\end{array}$ & $\begin{array}{l}\text { Mixed vertebral anom- } \\
\text { alies }(n=8)\end{array}$ & Total $(n=48)$ \\
\hline \multirow{2}{*}{$\begin{array}{l}\text { Single cord } \\
\text { anomaly } \\
(n=24)\end{array}$} & SCM & 8 & 6 & 3 & 17 \\
\hline & Syringomyelia & 4 & 3 & 0 & 7 \\
\hline \multirow{6}{*}{$\begin{array}{l}\text { Multiple cord } \\
\text { anomalies } \\
(n=24)\end{array}$} & Tethered cord ${ }^{\mathrm{a}}$ & 1 & 3 & 2 & 6 \\
\hline & SCM + Syringomyelia & 3 & 1 & 1 & 5 \\
\hline & $\mathrm{SCM}+$ tethered cord $^{\mathrm{a}}$ & 2 & 1 & 0 & 3 \\
\hline & $\mathrm{SCM}+$ intraspinal mass & 2 & 0 & 1 & 3 \\
\hline & Syrinx + tethered cord ${ }^{a}$ & 4 & 0 & 0 & 4 \\
\hline & 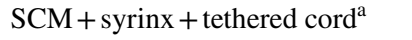 & 1 & 1 & 1 & 3 \\
\hline
\end{tabular}

${ }^{a}$ All cases of tethered cord had a low-lying conus. SCM-Split cord malformation

conus was at L3 in seven patients, L4 in six, at L5 in two and $\mathrm{S} 1$ in one patient.

Forty-one patients had 52 organ defects, out of which 15 were cardiac, 19 renal, 15 patients had limb anomalies and three had other abnormalities. Thirteen patients with organ defects had concomitant spinal cord anomalies. There was no significant difference in the frequency of cord anomalies between those with and without other organ defects. The frequency of other organ defects was similar in patients with failure of formation, failure of segmentation and mixed vertebral anomalies.

\section{Discussion}

Congenital spinal deformities are secondary to asymmetrical growth of the spine, due to underlying vertebral anomalies. The rate of curve progression depends on age of presentation, type and location of the abnormal vertebrae. The deformity progresses rapidly during the first 5 years of life and the adolescent growth spurt, which represent the periods of maximal spinal growth. In general, $25 \%$ of the curves do not progress, $25 \%$ of the curves progress slowly and $50 \%$ of the curves may show rapid progression [19]. In the current study, $31 \%$ of curves had minimal progression with MCPI of $<2^{\circ}, 47 \%$ had moderate progression with MCPI of $2^{\circ}-5^{\circ}$ and $22 \%$ of the curves showed rapid progression, with an MCPI $>5^{\circ}$. Some authors advocate early surgery in congenital scoliosis; however, these procedures are associated with higher complications such as surgical site infection, pseudarthrosis, implant failure, short trunk and crankshaft phenomenon [19]. Though non-operative treatment is not very effective in the management of congenital scoliosis, patients with anomalies known to have a low MCPI can be observed till 10 years of age to avoid these complications. However, rapidly progressive curves need to be operated earlier. In the current series, 29 patients were operated within 1 year of presentation and 126 patients were operated around the adolescent growth spurt.

The prevalence of intraspinal anomalies in congenital scoliosis ranges from 20 to $58 \%$ [3, 6-11]. In the current study, the frequency of intraspinal anomalies was $21.2 \%$. This is similar to the findings of McMaster et al. and Gandhari et al. [3, 8]. Gandhari et al. did not find any correlation between vertebral and cord anomalies, while Shen et al. and Basu et al. found a higher incidence of intraspinal anomalies in patients with failure of segmentation and mixed vertebral lesions [6-8]. In the current study, there was a significantly higher frequency of cord anomalies in patients with failure of segmentation. The presence of intraspinal anomalies may compound neurological complications, in patients with kyphosis, warranting earlier intervention (Fig. 1). Thus, MRI 


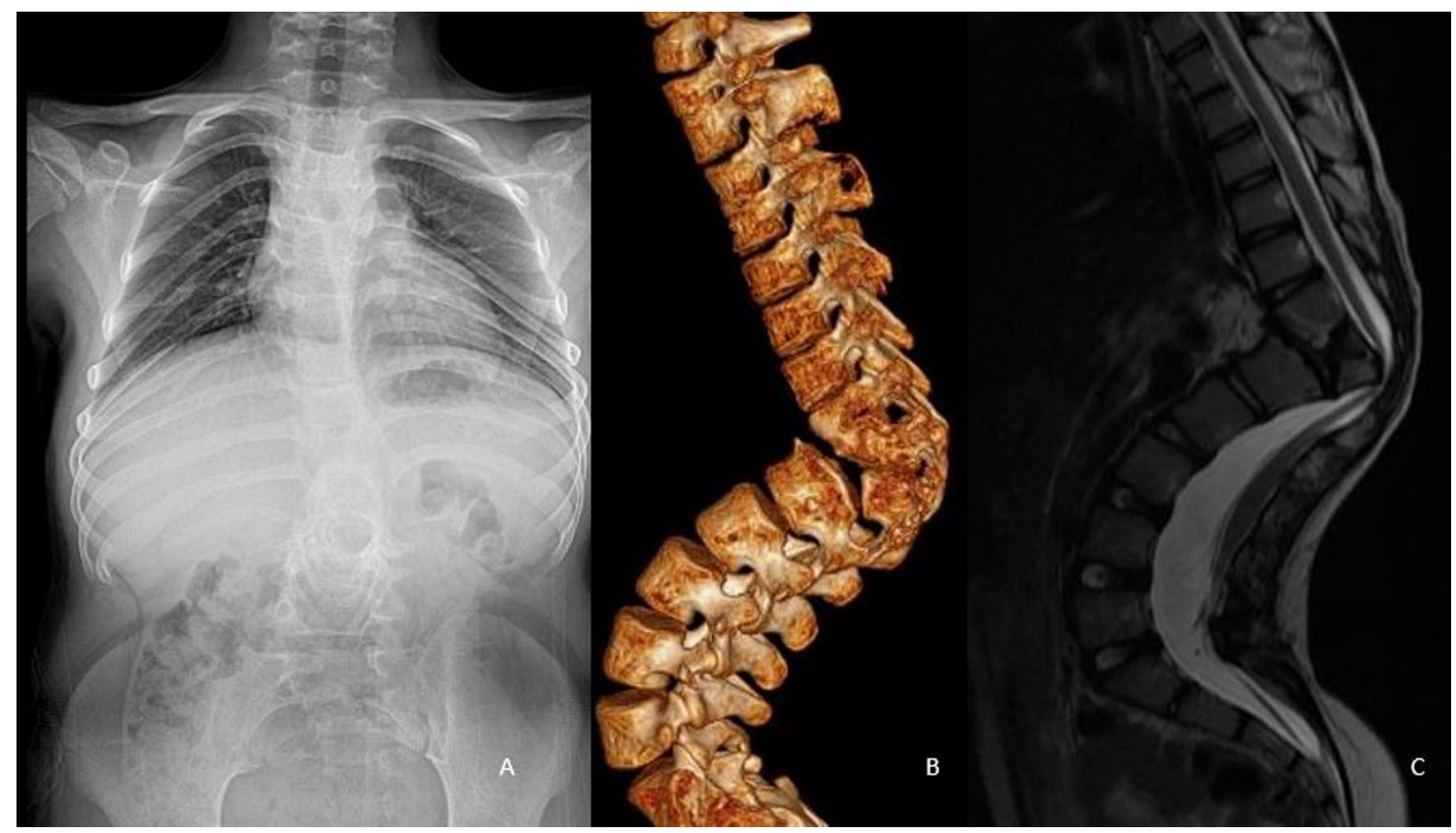

Fig. 1 a Preoperative radiograph of a 12-year-old girl showing spinal deformity. b Computed tomographic scan with three-dimensional reconstruction revealed kyphoscoliosis due to multiple hemivertebrae

of the spine is mandatory to rule out occult cord abnormalities prior to deformity correction. The timing of MRI depends on the clinical presentation of the patient with the congenital spinal deformity. The presence of neurological deficit and cutaneous markers of intraspinal anomalies in a child below the age of 5 years may warrant MRI under general anaesthesia. In the neurologically intact child with no features of cord anomalies, MRI can be deferred until the child is able to tolerate the investigation [7].

SCM was the most common intraspinal abnormality in the current study (64.6\%), followed by syringomyelia (39.6\%) and tethered cord with low-lying conus (33\%). This is similar to the findings of some investigators $[3,4,6,8$, 20]. In contrast, other studies reported tethered cord to be the most common intraspinal anomaly [7, 9-11]. In the present study, type 2 SCM was more common than type 1 SCM. Feng et al. observed that type $1 \mathrm{SCM}$ was more frequently associated with multiple, mixed vertebral anomalies [20]. However, in the current study, no such association was seen and type $2 \mathrm{SCM}$ was seen most frequently in patients with thoracic hemivertebrae. Although patients with SCM and neurological deficits require neurosurgical intervention, the management of asymptomatic SCM remains controversial. It is generally accepted that type $1 \mathrm{SCM}$ produces a tethering effect on the spinal cord, with growth, and must be addressed prior to deformity correction [19-21]. On the other hand, many authors reported that patients with type 2 SCM who underwent deformity correction without neurosurgical from D10 to D12, and c magnetic resonance imaging demonstrated compressive myelopathy and tethered cord with low-lying conus

intervention had no neurological complications on followup. Considering the complications associated with surgery for split cord, such as neurological deterioration, CSF leak and infection, these authors opined that in patients with type 2 SCM, deformity correction can be done safely without neurosurgical intervention [22-25]. In the current series, all patients with type $1 \mathrm{SCM}$ and none of the patients with type $2 \mathrm{SCM}$ underwent neurosurgical intervention, prior to deformity correction.

Syringomyelia is another common anomaly seen in congenital scoliosis. The progression of the deformity in congenital scoliosis is unrelated to the location and size of the syrinx, unlike scoliosis in patients with Chiari malformation and idiopathic syringomyelia. In patients with congenital scoliosis, indirect tethering at the site of SCM, tethered cord, or vertebral anomalies breaks down the lining membrane of the central canal resulting in cavitation and syrinx, whereas, in Chiari malformation, growth of the spinal column produces traction, resulting in caudal migration of the cerebellar tonsils. This alters the flow of cerebrospinal fluid resulting in a large syrinx, usually in the cervical spine. Hence, in patients with congenital scoliosis, surgical treatment of the syrinx is not necessary, unless it is associated with a Chiari malformation or neurological deficit [18, 26, 27]. In the current series, none of the patients had concomitant Chiari malformation; however, $63.2 \%$ of cases with syringomyelia had other associated cord anomalies (Fig. 2). The syrinx was small to medium in size and located mainly in the thoracic 


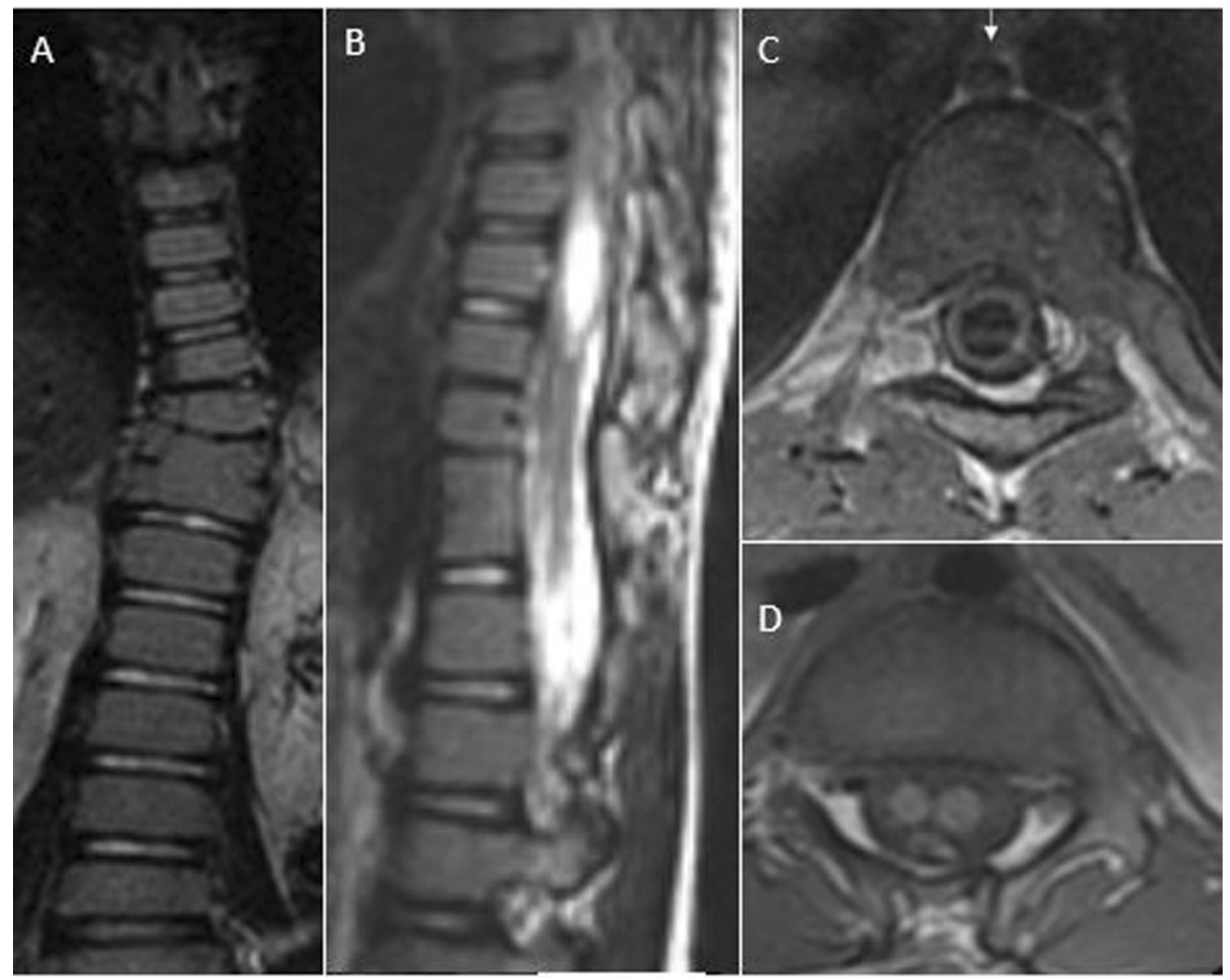

Fig. 2 Magnetic resonance imaging of a 6-year-old patient with congenital scoliosis showing a D11-D12 block vertebrae, b, c D6-D9 syringomyelia and $\mathbf{d}$ split cord malformation from D11 to L1 levels

and cervicothoracic region. This is similar to the findings of Ozerdemoglu et al. [18].

In the current study, tethered cord was the third most common cord anomaly with a frequency of $7 \%$. All cases of tethered cord were associated with low lying conus, and $62.5 \%$ had other concomitant cord anomalies. The presence of tethered cord in a case of congenital scoliosis increases the risk of neurological complications with growth and deformity correction. Thus, all cases of tethered cord must be closely observed and surgical detethering should be done if the patient develops neurological deficits or prior to deformity correction $[19,25]$. All cases of congenital scoliosis with tethered cord in the current series underwent surgical detethering early, to minimize the risk of neurological deterioration.

Some patients with congenital scoliosis and intraspinal anomalies require neurosurgical intervention for the cord anomalies, prior to or at the time of deformity correction, to prevent neurological complications. Conventionally, this is accomplished in two stages, with a gap of 3 to 6 months. The arguments in favour of a two-stage procedure are to ensure no neurological deficit occurred during the neurosurgical intervention and to allow adequate time for recovery $[3,5]$. Some authors have advocated both procedures be done in a single stage [21]. In the current series, all patients requiring neurosurgical intervention underwent two-stage procedure with a minimum gap of 3 months.

In the current study, the frequency of other organ anomalies was $18.1 \%$, while reports in the literature found the frequency to be $8-26 \%[4,6,7,20]$. The frequency of intraspinal abnormalities was similar in those with and without other organ anomalies. This is similar to the findings of Shen et al. [6]. The frequency of other organ defects was similar in patients with failure of formation, failure of segmentation and mixed vertebral anomalies. Active detection of asymptomatic underlying renal and cardiac abnormalities must be undertaken in all patients with congenital scoliosis.

The strengths of the current study include the large sample size and detailed analyses done on consecutive patients with congenital scoliosis irrespective of the type of treatment. The drawbacks include its retrospective nature and the pattern of rib anomalies not being assessed. While 
accurate identification of vertebral anomalies was assisted by the availability of the supine-stretch AP radiographs, in recent times, low-dose CT scans of the whole spine with $3 \mathrm{D}$ reconstruction have been advocated. It is useful in better visualization of the anterior and posterior vertebral abnormalities, especially in complex mixed vertebral lesions. It is also of great help in developing strategies for surgical treatment [28].

Acknowledgements Open access funding provided by Manipal Academy of Higher Education, Manipal.

\section{Compliance with ethical standards}

Conflict of interest The authors declare that they have no conflict of interest.

Ethical approval All procedures performed in studies involving human participants were in accordance with the ethical standards of the institutional and/or national research committee and with the 1964 Helsinki Declaration and its later amendments or comparable ethical standards. Institutional ethical clearance was obtained before starting the study.

Human and animal rights This article does not contain any studies with animals performed by any of the authors.

Informed consent Informed consent was obtained from all individual participants included in the study.

Open Access This article is licensed under a Creative Commons Attribution 4.0 International License, which permits use, sharing, adaptation, distribution and reproduction in any medium or format, as long as you give appropriate credit to the original author(s) and the source, provide a link to the Creative Commons licence, and indicate if changes were made. The images or other third party material in this article are included in the article's Creative Commons licence, unless indicated otherwise in a credit line to the material. If material is not included in the article's Creative Commons licence and your intended use is not permitted by statutory regulation or exceeds the permitted use, you will need to obtain permission directly from the copyright holder. To view a copy of this licence, visit http://creativecommons.org/licenses/by/4.0/.

\section{References}

1. Tsou PM, Yau AC, Hodgson AR (1980) Embryogenesis and prenatal development of congenital vertebral anomalies and their classification. Clin Orthop Relat Res 152:211-231

2. McMaster MJ, Ohtsuka K (1982) The natural history of congenital scoliosis. A study of two hundred and fifty-one patients. J Bone Jt Surg Am 64(8):1128-1147

3. McMaster MJ (1984) Occult intraspinal anomalies and congenital scoliosis. J Bone Jt Surg Am 66(4):588-601

4. Mohanty S, Kumar N (2000) Patterns of presentation of congenital scoliosis. J Orthop Surg 8(2):33-37

5. Winter RB (1973) Congenital scoliosis. Clin Orthop Relat Res 93:75-94

6. Shen J, Wang Z, Liu J, Xue X, Qiu G (2013) Abnormalities associated with congenital scoliosis: a retrospective study of $226 \mathrm{Chi}-$ nese surgical cases. Spine 38(10):814-818
7. Basu PS, Elsebaie H, Noordeen MH (2002) Congenital spinal deformity: a comprehensive assessment at presentation. Spine 27(20):2255-2259

8. Ghandhari H, Tari HV, Ameri E, Safari MB, Fouladi DF (2015) Vertebral, rib, and intraspinal anomalies in congenital scoliosis: a study on 202 Caucasians. Eur Spine J 24(7):1510-1521

9. Gupta N, Rajasekaran S, Balamurali G, Shetty A (2016) Vertebral and intraspinal anomalies in Indian population with congenital scoliosis: a study of 119 consecutive patients. Asian Spine J 10(2):276-281

10. Bradford DS, Heithoff KB, Cohen M (1991) Intraspinal abnormalities and congenital spine deformities: a radiographic and MRI study. J Pediatr Orthop 11(1):36-41

11. Blake NS, Lynch AS, Dowling FE (1986) Spinal cord abnormalities in congenital scoliosis. Ann Radiol Paris 29(3-4):377-379

12. Hedequist D, Emans J (2004) Congenital scoliosis. J Am Acad Orthop Surg 12(4):266-275

13. Cobb JR (1948) Outline for the study of scoliosis. Instr Course Lect AAOS 5:261-275

14. Upadhyay SS, Saji MJ, Sell P, Sell B, Yau AC (1994) Longitudinal changes in spinal deformity after anterior spinal surgery for tuberculosis of the spine in adults. A comparative analysis between radical and debridement surgery. Spine 19(5):542-549

15. Pang D, Dias MS, Ahab-Barmada M (1992) Split cord malformation: part I: a unified theory of embryogenesis for double spinal cord malformations. Neurosurgery 31(3):451-480

16. Pang D (1992) Split cord malformation: part II: clinical syndrome. Neurosurgery 31(3):481-500

17. Tokunaga M, Minami S, Isobe K, Moriya H, Kitahara H, Nakata Y (2001) Natural history of scoliosis in children with syringomyelia. J Bone Jt Surg Br 83(3):371-376

18. Özerdemoglu RA, Denis F, Transfeldt EE (2003) Scoliosis associated with syringomyelia: clinical and radiologic correlation. Spine 28(13):1410-1417

19. Pahys JM, Guille JT (2018) What's new in congenital scoliosis? J Pediatr Orthop 38(3):e172-e179

20. Feng F, Tan H, Li X, Chen C, Li Z, Zhang J, Shen J (2017) Radiographic characteristics in congenital scoliosis associated with split cord malformation: a retrospective study of 266 surgical cases. BMC Musculoskelet Disord 18(1):420-427

21. Hamzaoglu A, Ozturk C, Tezer M, Aydogan M, Sarier M, Talu U (2007) Simultaneous surgical treatment in congenital scoliosis and/or kyphosis associated with intraspinal abnormalities. Spine 32(25):2880-2884

22. Jamil M, Bunnister CM (1992) A report of children with spinal dysraphism managed conservatively. Eur J Pediatr Surg 2(1):26-28

23. Ayvaz M, Akalan N, Yazici M, Alanay A, Acaroglu RE (2009) Is it necessary to operate all split cord malformations before corrective surgery for patients with congenital spinal deformities? Spine 34(22):2413-2418

24. Shen J, Zhang J, Feng F, Wang Y, Qiu G, Li Z (2016) Corrective surgery for congenital scoliosis associated with split cord malformation: it may be safe to leave diastematomyelia untreated in patients with intact or stable neurological status. J Bone Jt Surg Am 98(11):926-936

25. Drake JM (2007) Surgical management of the tethered spinal cord-walking the fine line. Neurosurg Focus 23(2):e4

26. Tan H, Shen J, Feng F, Zhang J, Wang H, Chen C, Li Z (2018) Clinical manifestations and radiological characteristics in patients with idiopathic syringomyelia and scoliosis. Eur Spine J 27(9):2148-2155

27. Iskandar BJ, Oakes J, McLaughlin C, Osumi AK, Tien RD (1994) Terminal syringohydromyelia and occult spinal dysraphism. J Neurosurg 81(4):513-519 
28. Kawakami N, Tsuji T, Imagama S, Lenke LG, Puno RM, Kuklo TR, Spinal Deformity Study Group (2009) Classification of congenital scoliosis and kyphosis: a new approach to the three-dimensional classification for progressive vertebral anomalies requiring operative treatment. Spine 34(17):1756-1765
Publisher's Note Springer Nature remains neutral with regard to jurisdictional claims in published maps and institutional affiliations. 